\title{
Deep-learning-assisted detection and termination of spiral and broken-spiral waves in mathematical models for cardiac tissue
}

\author{
Mahesh Kumar Mulimani, ${ }^{*}$ Jaya Kumar Alageshan, ${ }^{\dagger}$ and Rahul Pandit ${ }^{\ddagger}$ \\ Centre for Condensed Matter Theory, Department of Physics, Indian Institute of Science, Bangalore 560012, India
}

(Received 16 May 2019; accepted 15 April 2020; published 11 May 2020)

\begin{abstract}
Unbroken- and broken-spiral waves, in partial-differential-equation (PDE) models for cardiac tissue, are the mathematical analogs of life-threatening cardiac arrhythmias, namely, ventricular tachycardia and ventricularfibrillation. We develop (a) a deep-learning method for the detection of unbroken- and broken-spiral waves and (b) the elimination of such waves, e.g., by the application of low-amplitude control currents in the cardiac-tissue context. Our method is based on a convolutional neural network (CNN) that we train to distinguish between patterns with spiral-waves $\mathscr{S}$ and without spiral-waves $\mathscr{N} \mathscr{S}$. We obtain these patterns by carrying out extensive direct numerical simulations of PDE models for cardiac tissue in which the transmembrane potential $V$, when portrayed via pseudocolor plots, displays patterns of electrical activation of types $\mathscr{S}$ and $\mathscr{N} \mathscr{S}$. We then utilize our trained CNN to obtain, for a given pseudocolor image of $V$, a heatmap that has high intensity in the regions where this image shows the cores of spiral waves and the associated wavefronts. Given this heatmap, we show how to apply low-amplitude currents of a two-dimensional Gaussian profile to eliminate spiral-waves efficiently. Our in silico results are of direct relevance to the detection and elimination of these arrhythmias because our elimination of unbroken or broken-spiral waves is the mathematical analog of low-amplitude defibrillation.
\end{abstract}

DOI: 10.1103/PhysRevResearch.2.023155

\section{INTRODUCTION}

The normal pumping of blood by mammalian hearts is initiated by electrical waves of excitation that propagate through cardiac tissue and induce cardiac contractions. The abnormal propagation of such waves can lead to cardiac arrhythmias, such as ventricular tachycardia (VT) and ventricular fibrillation (VF), which cause sudden cardiac death (SCD), which is among the leading causes of death in the industrialized world [1-3] (see, e.g., Refs. [4,5] for SCD data from India and the U.S.). The principal cause of VT and VF are spiral or scroll waves of electrical activation in cardiac tissue; unbroken (broken) spiral or scroll waves are associated with VT (VF) [6-8]. Such waves have been studied, e.g., in ex vivo [9-11] and in vivo [12-14] in mammalian hearts, in vitro $[6-8,15]$ in cultures of cardiac myocytes, and in silico [16-18] in mathematical models for cardiac tissue. The efficient elimination of such spiral or scroll waves and the subsequent restoration of the normal rhythm of a mammalian heart is a difficult problem; this can be attempted by pharmacological means [19] or by electrical means called defibrillation [20]. Defibrillation by the application of low-amplitude current pulses

\footnotetext{
*maheshk@iisc.ac.in

†jayaka@iisc.ac.in

${ }^{\ddagger}$ rahul@iisc.ac.in; also at Jawaharlal Nehru Centre For Advanced Scientific Research, Jakkur, Bangalore, India.
}

Published by the American Physical Society under the terms of the Creative Commons Attribution 4.0 International license. Further distribution of this work must maintain attribution to the author(s) and the published article's title, journal citation, and DOI. is the grand challenge here [21]. Two important steps are required for such defibrillation: (a) An efficient detection of spiral waves or their broken-wave forms; (b) the elimination of such waves by low-amplitude electrical pulses [16-18,20] or through optogenetic methods [22].

We develop a deep-learning method, based on a convolutional neural network (CNN), that helps us to accomplish task (a). We then develop the mathematical analog of a defibrillation scheme for the efficient elimination of wellformed spiral and broken-spiral waves in two dimensions (2D). Electrical waves in cardiac tissue belong to a large class of nonlinear waves in excitable media, e.g., calcium-ion waves in Xenopus oocytes [23], waves in chemical reactions of the Belousov-Zhabotinsky type [24], waves that occur during the oxidation of carbon monoxide on the surface of platinum [25-27], excitable-wave patterns in a recent semiconductorlaser experiment [28], and waves in Dictyostelium discoideum that are associated with cyclic-AMP signaling [29,30]; our step (a) can be applied, mutatis mutandis, for the detection of spiral waves in such systems.

Specifically, we train our CNN to classify, into the following two sets, patterns of electrical-wave activation, which we obtain from in silico studies of different mathematical models for cardiac tissue [31-35]: (a) spiral waves $(\mathscr{S})$ and (b) no spiral waves $(\mathscr{N} \mathscr{S})$ (Fig. 1). Next, we use our trained CNN to detect spiral-wave patterns, with both unbroken and broken spirals. We then use the outputs from our CNN to construct a heatmap that has a high intensity in the regions with spiral cores. We demonstrate how to eliminate the broken or unbroken spiral waves by applying low-amplitude current stimuli at those positions at which the heatmap has high intensity; this is the mathematical analog of defibrillation [20]. 


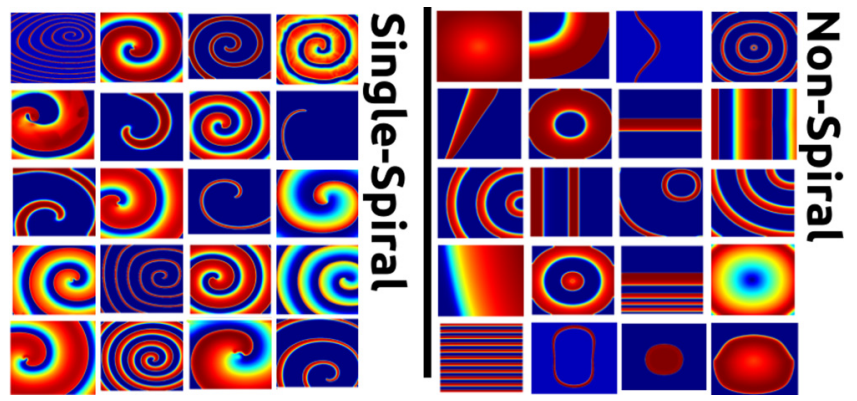

FIG. 1. Collages of illustrative pseudocolor plots of $V$ with (a) single-spiral $(\mathscr{S})$ images and (b) no spiral $(\mathscr{N} \mathscr{S})$ images, which we use for training our $\mathrm{CNN}$.

\section{MODELS, METHODS, AND RESULTS}

Mathematical models for cardiac tissue use nonlinear partial differential equations (PDEs) of the reaction-diffusion type:

$$
\begin{array}{cl}
\frac{\partial V}{\partial t}=D_{0} \nabla^{2} V+f(V, g)+I_{\mathrm{ext}}, & \frac{\partial g}{\partial t}=\epsilon(V, g) h(V, g), \\
\frac{\partial V}{\partial t}=D_{0} \nabla^{2} V-\frac{I_{\text {ion }}}{C_{m}}+I_{\text {ext }}, & I_{\text {ion }}=\sum_{i} I_{i},
\end{array}
$$

where $V, I_{i}, I_{\text {ext }}$, and $C_{m}$ are the transmembrane potential, the ionic current for ion channel $i$, the external current, and the membrane capacitance, respectively (see [36] for the forms of $f, h$, and $I_{i}$ in these models). For our direct numerical simulation (DNS), we use two classes of models, namely (a) two-variable models [Eq. (1)] such as the Barkley model [31] and the Aliev-Panfilov model [32], and (b) biologically realistic models with ion channels, ion pumps, and ion exchangers [Eq. (2)], such as the Luo-Rudy phase-I model (LR-I) [33], the Tusscher-Panfilov model (TP06) [34], and the O'Hara-Rudy model (ORd) [35] (see [36] for further details). We focus on electrical activity in cardiac tissue, and our DNS yields patterns such as spiral waves, target waves, and plane waves [see Figs. 1(a) and 1(b)], and states with broken-spiral waves (Fig. 3). The details of our numerical simulations and the wide range of parameter we use are given in [36].

We use 22000 such images to train, and then test, our CNN. We create additional images by performing geometrical operations on the primary pseudocolor images $V$, e.g., inequivalent reflections about the horizontal and vertical axes, so that our dataset of images is not biased in favor of any particular orientation; this improves the training performance of our CNN. We train our CNN with $70 \%$ of the total number of images, and we save the remaining $30 \%$ of the images for the validation of our CNN model.

Our solutions of Eqs. (1) or (2) yield $V$ at $N^{2}$ grid points. We first define the normalized transmembrane potential $\tilde{V}=$ $\left(V-V_{\min }\right) /\left(V_{\max }-V_{\min }\right) ; V_{\max }$ and $V_{\min }$ are, respectively, the maximal and minimal values of $V$, so $0 \leqslant \tilde{V} \leqslant 1$. We then reduce a large number of grid points by specifying $\tilde{V}$ on $32 \times$ 32 points by using the resize function in MATLAB R2018b. We use the Deep Learning Toolbox in MATLAB R2018b to develop our CNN, which we depict schematically in Fig. 2. It has three main layers: (i) Input, (ii) Middle, and (iii) Final. The middle layer contains three sets of Convolution, Rectified Linear Unit (ReLU), and MaxPool sublayers. The final layer contains two fully connected artificial neural networks (ANNs). We give a brief description of the implementation of our CNN in [36].

We begin the training by feeding the image of $\tilde{V}$ to our $\mathrm{CNN}$. If the CNN output predicts the class of the input image incorrectly, then we use a cost function to rectify this error iteratively (until the $\mathrm{CNN}$ yields the correct output class). Specifically, we achieve this for our CNN by minimizing the cross-entropy cost function,

$$
\mathcal{C}=-\sum_{\ell=1}^{M} \sum_{q=0}^{\eta-1} \frac{\left[O_{\ell, q} \ln \left(\tilde{O}_{\ell, q}\right)+\left(1-O_{\ell, q}\right) \ln \left(1-\tilde{O}_{\ell, q}\right)\right]}{M}
$$

by using the stochastic-gradient-descent method with a learning rate $\alpha=0.001$ (see, e.g., Chap. 2 of Ref. [37]); here, $\tilde{O}_{\ell, q}$ are the CNN outputs $\left[\tilde{O}_{\ell, q} \in(0,1)\right]$ and $O_{\ell, q}$ are the real outputs for the input image $\ell$, and $M$ is the minibatch size (the total number of images is divided into subsets, called minibatches, with $M$ images each); we use $M=128$. For the class $\mathscr{S}, O_{\ell, 0}=1$ and $O_{\ell, 1}=0$; and for $\mathscr{N} \mathscr{S}, O_{\ell, 0}=0$ and $O_{\ell, 1}=1$.

A minimal spatial resolution is required to map any activation patterns in real optical imaging (see, e.g., Ref. [38]). Similarly, when we train our CNN, we find that the resized training image should have at least $32 \times 32$ points (on a square lattice with lattice spacing $0.8 \mathrm{~cm}$ ); e.g., if we use a resized image with $16 \times 16$ points, then we cannot distinguish spiral patterns clearly, as we illustrate in Fig. S2 in [36]. The authors of Ref. [38] use a grid of electrodes to detect simple spiral- and target-wave patterns; the minimal spacing that they require between these electrodes is $1 \mathrm{~cm}$, which is comparable to the lattice spacing of our $32 \times 32$ resized image.

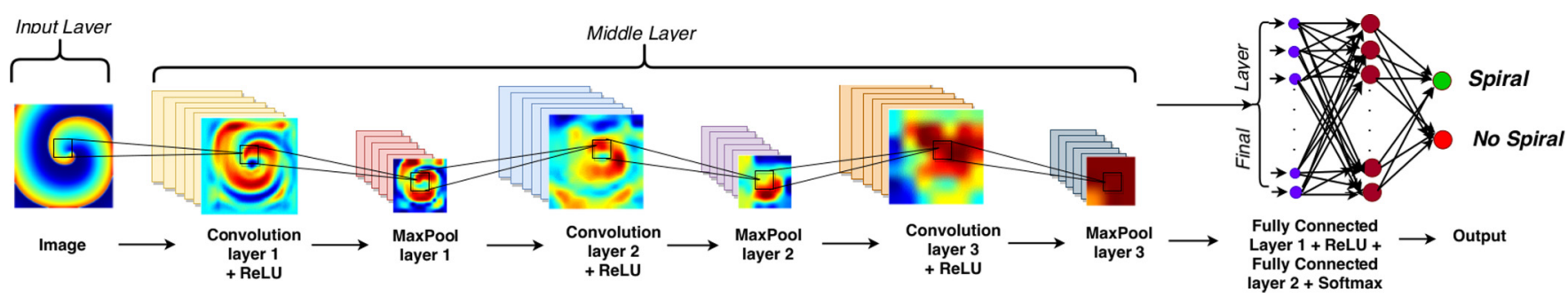

FIG. 2. A schematic diagram of our CNN showing the Input, Middle, and Final layers; the details of each one of these layer are given in the supplemental material [36]. 


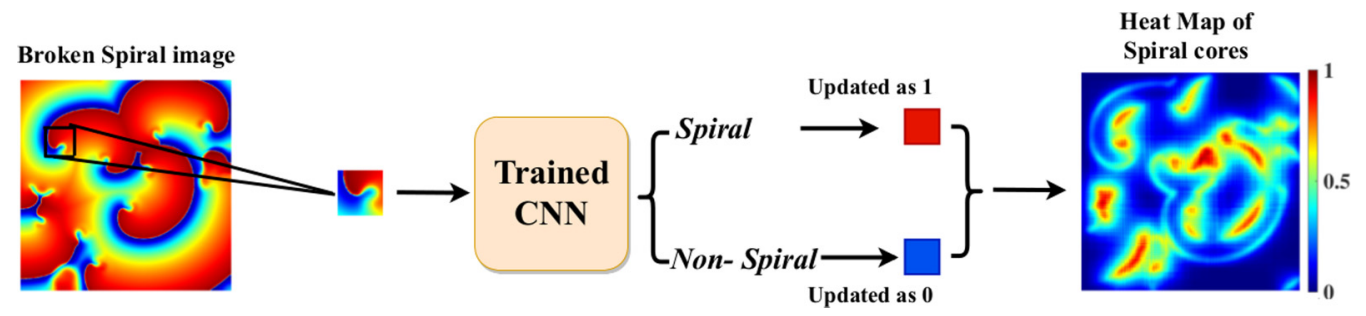

FIG. 3. Pseudocolor plots of (left panel) $V$, showing broken-spiral waves, and (right panel) the heatmap $\mathcal{H}$ for the image in the left panel.

Even though we train our CNN with single spiral-wave patterns, it manages to identify patterns with broken-spiral waves as belonging to the class $\mathscr{S}$ : We have checked that this CNN classifies 10000 broken-spiral wave patterns (see the pseudocolor plot of $V$ in Fig. 3) as $\mathscr{S}$, with an accuracy of $99.6 \%$. This is especially useful when we carry out the mathematical analog of defibrillation, i.e., the elimination of all spirals, unbroken or broken. We can indeed utilize our trained CNN to examine pseudocolor plots of $V$ during our numerical simulation of a mathematical model for cardiac tissue; the moment this CNN detects a pattern of type $\mathscr{S}$, we can eliminate it by the application of suitable currents. Here, we discuss a scheme for eliminating both broken and unbroken spiral waves; this relies on developing a heatmap, from a pseudocolor plot of $V$, which is identified by our CNN to lie in the class $\mathscr{S}$. The heatmap is the normalized sum of the outputs of our $\mathrm{CNN}$; the inputs to this $\mathrm{CNN}$ are multiple subimages of different sizes at different positions. We obtained these inputs from a given image (see Fig. 3). Mathematically, we define the heatmap as

$$
\mathcal{H}(i, j)=\frac{N_{p}}{N} \sum_{r=1}^{N / N p} \operatorname{CNN}\left(\chi_{i, j}^{r}\{V\}\right),
$$

$\forall i, j \in\{1,2, \ldots, N\}$; for the images used, $N=512$ grid points and $N_{p}=32$ grid points.

$\chi_{i, j}^{r}\{V\}$ is a standard matrix resizing function in MATLAB. The argument of the matrix-resizing function is a square matrix $V$ of size $32 r$ centered at the point $(i, j)$, with $1 \leqslant$ $i, j \leqslant N$ and $1 \leqslant r \leqslant\left(N / N_{p}\right)$.

The resized $\chi_{i, j}^{r}$, an image with $N_{p} \times N_{p}$ pixels, is the input into our CNN (Fig. 2), and its output, 0 (for $\mathscr{N} \mathscr{S}$ ) or 1 (for $\mathscr{S})$, is summed over $r$ to obtain $\mathcal{H}(i, j)$ for a given input pseudocolor plot of $V$. Clearly, $\mathcal{H}(i, j) \in[0,1]$, and it is large if there is a spiral core near the point $(i, j)$. In the left and right panels of Fig. 3 we show a broken-spiral-wave pattern, and the corresponding heatmap.

Our heatmap method is based on the detection of spiral cores by our trained CNN. Therefore, we compare our heatmap method with earlier works on phase-singularitydetection studies in optical images from ex vivo $[9,10]$ and in vivo $[39,40]$. The above-cited experimental studies obtain activation maps, phase maps, and APD- and CV-distribution maps, and they also detect phase singularities (PSs) in activation patterns by using fluorescence data; defibrillation strategies are not their goal. The training of our CNN also requires similar images (we use pseudocolor images of $V_{m}$ ). PS detection is not the main goal of our study; we concentrate on using our CNN to develop a heatmap, which we then use to develop a low-amplitude defibrillation scheme.

Optical images in experimental studies are noisy (Refs. [9,10,41]). We discuss here the performance of our trained CNN on the experimental data. We have checked that our trained $\mathrm{CNN}$ successfully generates heatmaps of the experimental images in Refs. [41-44]. The experimental image data are noisy and unclear about the type of patterns present (see, for example, Fig. 8 in [10] and Fig. 1 in [41]). For example, in Figs. 8(e) and 8(f) of Ref. [10], one of the spirals is clearly apparent, but it is not clear whether the other patterns are spirals or other excitations. This difficulty can be overcome by performing wavelet denoising on the image and by using Gaussian smoothening filters. After performing wavelet denoising [41] and applying a Gaussian filter, the number of spirals is clearly apparent (we have not shown this here). Such methods also work for the noisy image in Fig. 1 of Ref. [41]. We do not have access to raw experimental data; therefore, we have mimicked experimental noisy images by using data from our simulations, and we have then used our trained $\mathrm{CNN}$ to generate the heatmap. In Fig. 4(a) we present an image from our simulations; Fig. 4(b) shows the same image after we have added noise [with a signal-to-noise ratio $(\mathrm{SNR})=0.369$ ] by randomly picking grid points and replacing the values of $V_{m}$ to $-86 \mathrm{mV}$ using uniform distribution to mimic the experimental data.

The noisy image in Fig. 4(b) mimics the noisy experimental image of Fig. 1 of Ref. [41]. We smoothen this noisy image by using a Gaussian filter; we then feed the output to our trained CNN to generate the heatmap that is shown in Fig. 5. Figure 6 shows the noisy images for different values of the SNR and their corresponding heatmaps, which are generated by our trained CNN. (a) Image

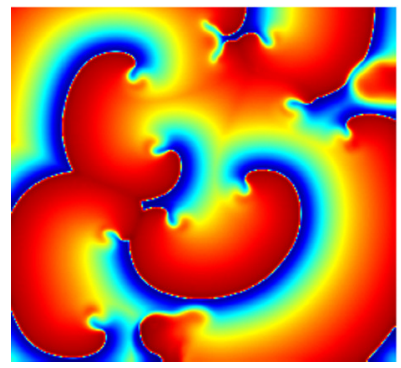

(b) Noisy Image $(\mathrm{SNR}=0.369)$

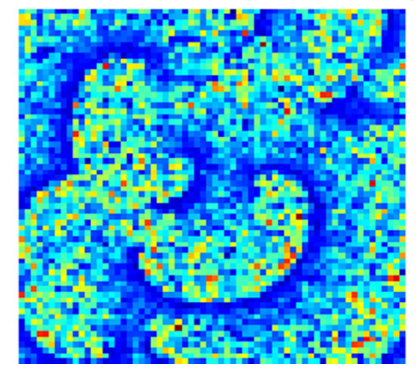

FIG. 4. (a) Image from simulation data, (b) noisy image obtained from simulation data. 


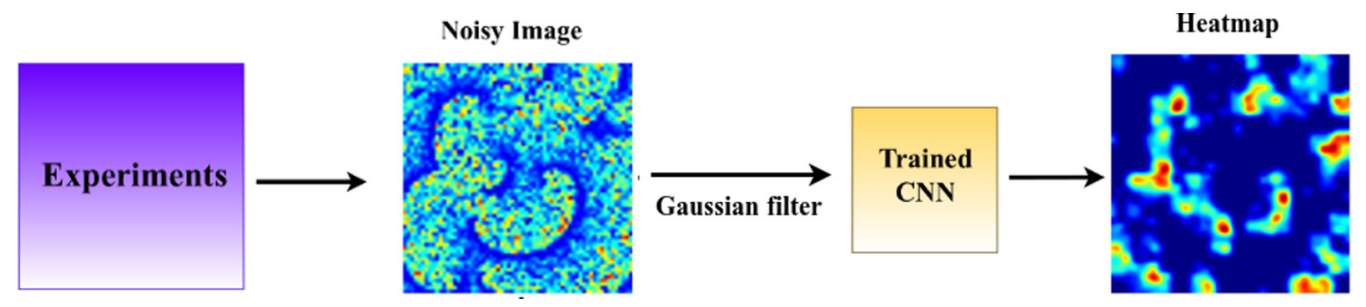

FIG. 5. Schematic diagram showing the process of generating a heatmap for a noisy image. The SNR value of a noisy image in this particular case is $\mathrm{SNR}=0.369$.

We discuss below the fundamental idea of our defibrillation scheme. Spiral-wave excitations emanate from the spiral core, so we might expect that the elimination of this core could lead to the removal of the spiral-wave. However, when we apply a current pulse on a disk centered at the spiral core, we find that it leads to the formation of multiple spiral cores along the boundary of the disk. We prevent the formation of such multiple spiral cores by applying a current pulse with a spatial profile that is a symmetrical, 2D Gaussian (centered at the spiral core, with equal widths in both the $x$ and $y$ directions, $\sigma_{x}=\sigma_{y}=\sigma$, and with a peak intensity of $I_{\mathrm{def}}$ ), and we can remove the core and the wavefronts without forming multiple spiral cores (Fig. 7). We refer to such a current profile as a 2D-Gaussian current with width $\sigma$ or simply as a Gaussian current.

We note that our defibrillation scheme does not use pacing protocols (of the type that are used, e.g., in the antitachycardia-pacing protocol of Refs. [45,46]).

Our heatmap can be used to eliminate broken-spiral waves. We now consider a pattern with broken-spiral waves (see the left panel of Fig. 3), where we observe multiple spiral waves with different (spiral-arm) wavelengths. We have mentioned above that a single spiral wave can be eliminated by the application of a 2D-Gaussian current profile. Therefore, for many broken-spiral waves, we require multiple Gaussian-profile currents to eliminate these waves, hence we construct a lattice of Gaussians, which we define as follows: On a square lattice of points, labeled by $\left(i^{\prime}, j^{\prime}\right)$, with $0 \leqslant i^{\prime}, j^{\prime} \leqslant N_{G}$, i.e., the side of the unit cell $a=N_{G} \Delta x(\mathrm{~cm})$, we impose a Gaussian $G\left(i^{\prime}, j^{\prime}\right)$ of width $\sigma$; the total, normalized contribution of these pulses at the point $(i, j)$, with $0 \leqslant i, j \leqslant(N-1)$, in the original image is

$$
\begin{gathered}
\tilde{\mathcal{G}}(i, j)=\sum_{l, m=0}^{N_{G}} G\left(i-l \frac{N}{N_{G}}, j-m \frac{N}{N_{G}}\right), \\
\mathcal{G}(i, j)=\tilde{\mathcal{G}}(i, j) / \tilde{\mathcal{G}}_{\max } ; \\
\tilde{\mathcal{G}}_{\max }=\max _{(i, j)}[\tilde{\mathcal{G}}(i, j)], \\
G(i, j)=\exp \left(\frac{-\left(i^{2}+j^{2}\right)}{\sigma^{2}}\right) .
\end{gathered}
$$

This lattice of Gaussians can be considered to be an array of contact electrodes that apply local currents. The heatmap $\mathcal{H}$ determines which electrodes have to be activated to apply local currents and the strengths of these currents. The external defibrillation current that we apply, $I_{\mathrm{ext}}$, for a time $t_{\mathrm{def}} \mathrm{ms}$ and with amplitude $I_{\text {def }}$, is

$$
I_{\text {ext }}=I_{\text {def }}(\mathcal{H} o \mathcal{G}) \text {. }
$$

The symbol " $O$ " denotes the Hadamard product, which is the element-wise multiplication of two matrices. Multiple spiral waves are eliminated by the application of external defibrillation current $I_{\text {ext }}$ (henceforth, the Gaussian-control scheme) in Eqs. (1) and (2).

In Figs. 8(a) and 8(b), we show, respectively, $V$ and its heatmap $\mathcal{H}$ [Eq. (4)] for an image with a broken-spiral wave in the TP06 model. Figures 8(c) and 8(d) depict, respectively, the summed and normalized $2 \mathrm{D}$ Gaussians $[\mathcal{G}$ in Eq. (6)] and the external defibrillation current $I_{\text {ext }}$ [with $I_{\text {def }}=5 \mathrm{pA} / \mathrm{pF}$
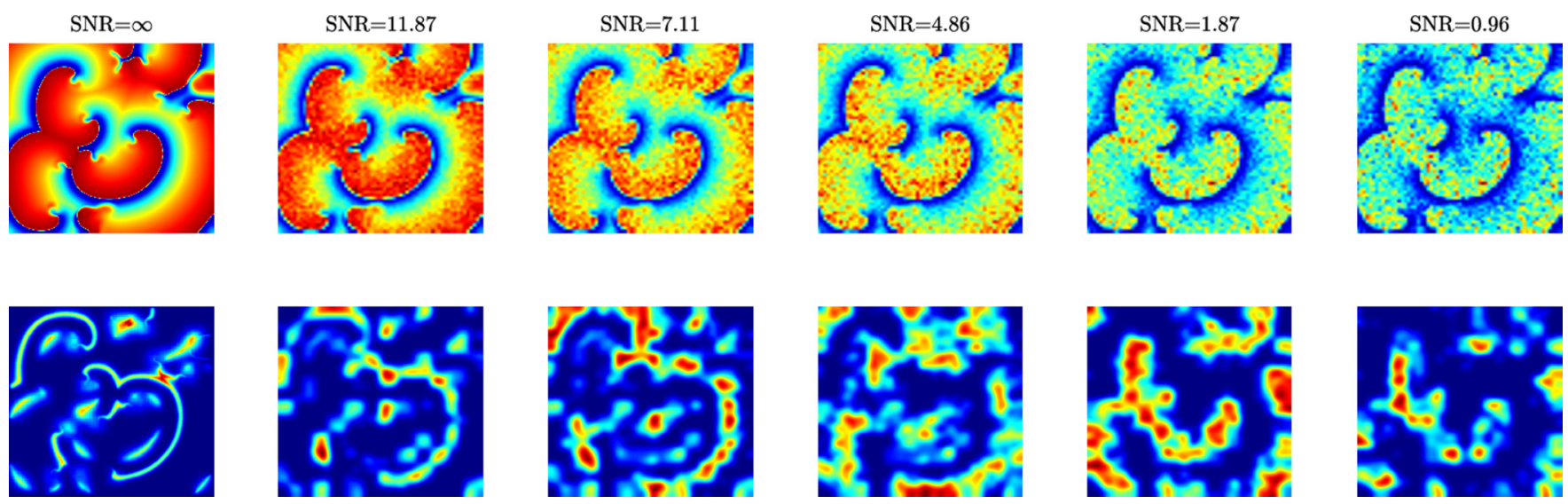

FIG. 6. Top row: Images of $V_{m}$ with added noise and different values of the SNR. Bottom row: The corresponding heatmaps generated from our trained CNN. 
(a)

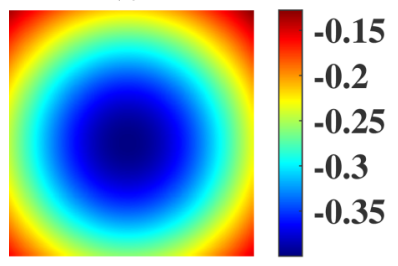

(b)

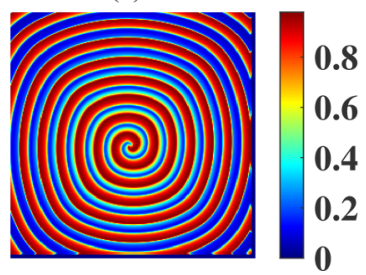

(c)

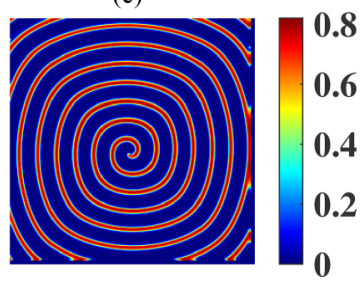

(d)

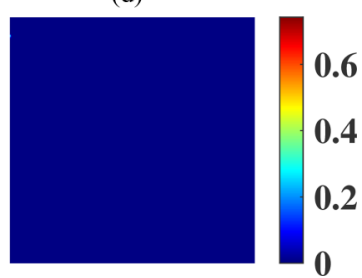

FIG. 7. Pseudocolor plots that show (a) the defibrillation current as $I_{\text {ext }}$ (single 2D-Gaussian centered at the spiral core), whose $I_{\text {def }}=0.5$ $\mathrm{pA} / \mathrm{pF}, \sigma=0.375 N \Delta x(\mathrm{~cm})$, and $N=256$. (b)-(d) $V$ of the Aliev-Panfilov model (Ref. [32]). (b) The spiral wave at the time of application, and (c),(d) after the defibrillation current is applied.

in Eq. (7)]. Our Gaussian-control scheme is illustrated in Figs. 8(e)-8(h); these show the spatiotemporal evolution of $V$ after the application of $I_{\mathrm{ext}}$, which is turned off at $t=t_{\mathrm{def}}$ (for the complete spatiotemporal evolution, see the video V1 in [36]). In Figs. 8(i)-8(1) we show the counterparts of Figs. 8(e)-8(h) for the control scheme in which a current pulse is applied on a square mesh to eliminate broken-spiral waves (Ref. [20] and the video V2 in [36]); we refer to this as the mesh-control scheme.

We now discuss the mechanism underlying the elimination of pathological waves of electrical activation by our defibrillation scheme. We have already explained the fundamental idea behind the elimination of a single spiral wave. To eliminate broken-spiral waves, we build on the idea of single spiral- wave elimination, but we must go beyond this because we also need the locations of the small spiral waves and their associated wavefronts, which can engender new excitations or spirals in the next instant of time (for examples, see the regions with wavefronts, which we have encircled by black lines in Fig. S6 of the supplemental material [36]). Hence, the elimination of spiral cores must also accompany the elimination of wavefronts, and we should target both the spiral cores and the wavefronts. If certain wavefronts yield nonzero values in the heatmap, then the local currents, which are applied along these wavefronts, make the adjoining regions refractory and stop incoming excitations. We observe in Figs. 3 and 8 (and also Fig. S4 in [36]) that our trained CNN not only detects spiral cores, but it also detects the wavefronts that yield (a)

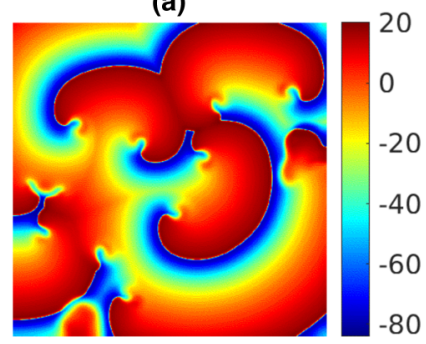

(e) $\mathrm{t}=40 \mathrm{~ms}$

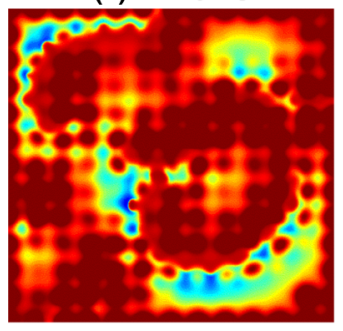

(i) $\mathbf{t}=\mathbf{0 m s}$

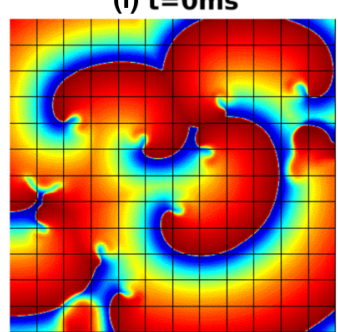

(b)

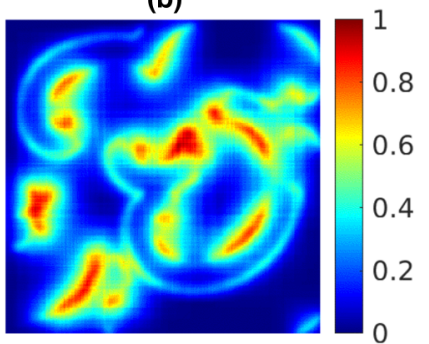

(f) $\mathrm{t}=60 \mathrm{~ms}$
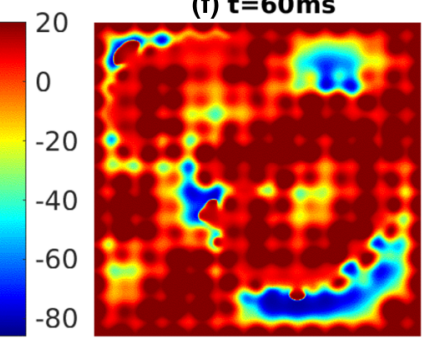

(j) $\mathrm{t}=40 \mathrm{~ms}$
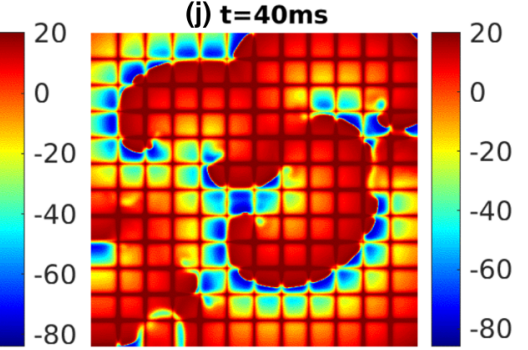

(c)

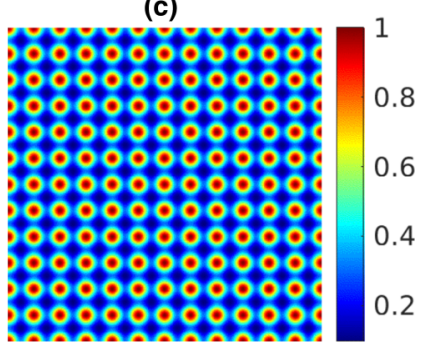

(g) $t=220 \mathrm{~ms}$
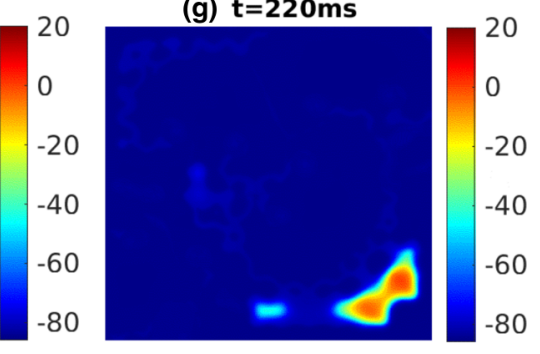

(k) $t=200 \mathrm{~ms}$

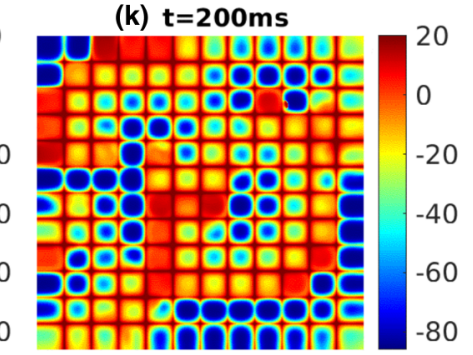

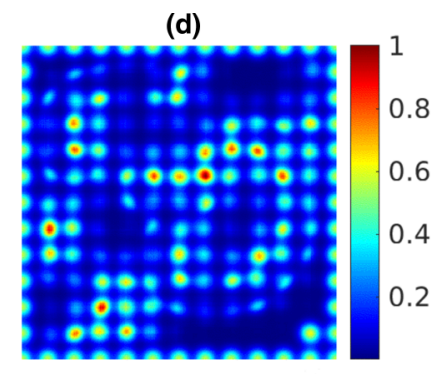

(h) $t=380 \mathrm{~ms}$

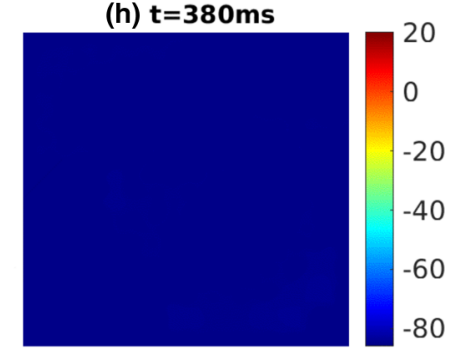

(I) $\mathrm{t}=360 \mathrm{~ms}$

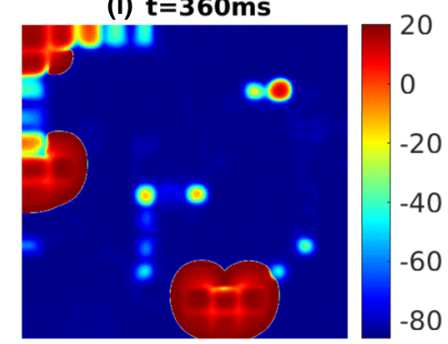

FIG. 8. Pseudocolor plots of (a) $V$ of a broken-spiral wave, (b) the corresponding heatmap $\mathcal{H}$ [Eq. (4)], (c) the summed and normalized 2D Gaussians $\left[\mathcal{G}(i, j)\right.$ in Eq. (6)], and (d) the Hadamard product $I_{\mathrm{ext}}(i, j)$ [with $I_{\text {def }}=5 \mathrm{pA} / \mathrm{pF}$ in Eq. (7)]. (e)-(h) Pseudocolor plots of $V$, at different representative times, showing the elimination of the broken spiral waves after the application of $I_{\mathrm{ext}}(i, j)$ for a time of $t=t_{\mathrm{def}}=120$ ms. (i)-(l) The analogs of (e)-(h) for the current-mesh control scheme of Ref. [20] (see the videos V1 and V2 in [36]). 
TABLE I. The parameter values that we use for our Gaussiancontrol (GC1 and GC2) and mesh-control (MC) schemes in our illustrative simulations.

\begin{tabular}{lccccc}
\hline \hline $\begin{array}{l}\text { Control } \\
\text { scheme }\end{array}$ & $N_{G}$ & $\begin{array}{c}a \\
(\mathrm{~cm})\end{array}$ & $\begin{array}{c}\sigma \\
(\mathrm{cm})\end{array}$ & $\begin{array}{c}I_{\text {def }} \\
(\mathrm{pA} / \mathrm{pF})\end{array}$ & $\begin{array}{c}t_{\text {def }} \\
(\mathrm{ms})\end{array}$ \\
\hline $\mathrm{GC} 1$ & 64 & 1.6 & $0.37 a$ & 5 & 120 \\
$\mathrm{GC} 2$ & 96 & 2.4 & $0.37 a$ & 5 & 120 \\
$\mathrm{MC}$ & 64 & & & 15 & 120 \\
\hline \hline
\end{tabular}

nonzero values in the heatmap. Hence the detection, by our CNN, of wavefronts that belong to $\mathscr{S}$ helps our defibrillation scheme.

The efficacy of our Gaussian-control scheme depends on $a, \sigma, I_{\mathrm{def}}$, and $t_{\mathrm{def}}$. We list these parameters (Table I) for two illustrative Gaussian-control runs- GC1 and GC2-and one run, $\mathrm{MC}$, in which we use a mesh-control scheme for the TP06 model. By comparing the results of such runs, we find that, for large values of $a$, our Gaussian-control scheme is not successful in removing spiral waves; e.g., in the TP06 model, broken-spiral waves are suppressed for the value of $a$ that we use in run GC1, but not for the value of $a$ in run GC2 (Table I). For the parameters in run GC1, Figs. 9(a)-9(d) show phase diagrams in the $\left(t_{\mathrm{def}}, \sigma\right)$ plane with fixed lattice constant $a=1.6 \mathrm{~cm}$ and for representative values of $I_{\mathrm{def}}$, with parameter regions in which our Gaussian-control scheme succeeds (red) and does not succeed (blue) in controlling broken-spiral waves. Furthermore, we show in Figs. 9(e)-9(h) the phase diagrams in the $\left(t_{\mathrm{def}}, a\right)$ plane for different values of $\sigma$ with fixed $I_{\text {def }}=10(\mathrm{pA} / \mathrm{pF})$. We observe that, for high values of lattice constant $a$, the scheme fails to eliminate the broken-spiral waves. Nonetheless, we observe that there is a regime of parameters where the scheme is successful for the given broken-spiral pattern. This Gaussian-control scheme also eliminates broken and unbroken-spiral waves in all the other cardiac-tissue models that we have studied (see Figs. S3 and S4 in [36]).

By comparing rows two and three of Fig. 8, we contrast the effectiveness of our Gaussian-control scheme with that of the mesh-control scheme of Ref. [20]. We find that our Gaussiancontrol scheme eliminates broken-spiral waves with $I_{\text {def }}=5$ $\mathrm{pA} / \mathrm{pF}$; by contrast, the mesh-control scheme requires $I_{\mathrm{def}}=$ $15 \mathrm{pA} / \mathrm{pF}$ for such elimination. Thus, the Gaussian-control scheme leads to the elimination of spiral waves with lower local currents than the mesh-control scheme, with all other parameters held fixed.

Another method that is used for the control of spiral waves is anti-tachycardia-pacing (ATP). Consider, e.g., the ATP of Refs. [45,46]; this technique is not always successful in eliminating spiral waves (see Refs. [45-47]). The pacing frequency should also be greater than the spiral-wave frequency [47]. This technique is mainly applicable for the unpinning of spirals attached to an obstacle, and this method is inadequate for the removal of high-frequency waves and spatiotemporally chaotic patterns; by contrast, our method is successful in the elimination of all such waves.

\section{CONCLUSIONS}

Our method of defibrillation using $\mathrm{CNN}$ uses the image; therefore, the detection of spiral waves is crucial for the success of our defibrillation scheme. We have checked that our CNN can be used to detect scroll waves in three-dimensional (3D) simulation domains (see Fig. S5 in [36]). The elimination of such $3 \mathrm{D}$ scroll waves by the application of currents on a

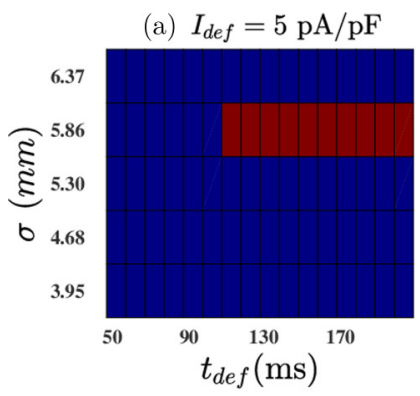

(e) $\sigma=0.59(\mathrm{~cm})$

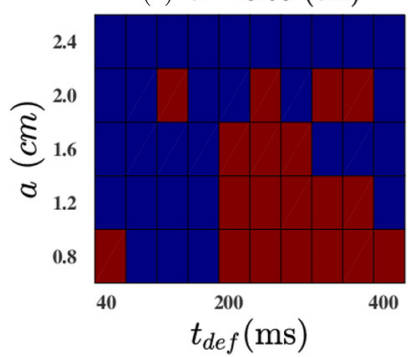

(b) $I_{\text {def }}=10 \mathrm{pA} / \mathrm{pF}$

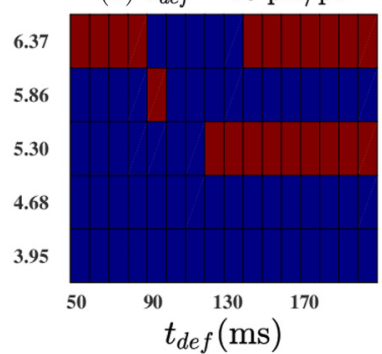

(f) $\sigma=0.64(\mathrm{~cm})$

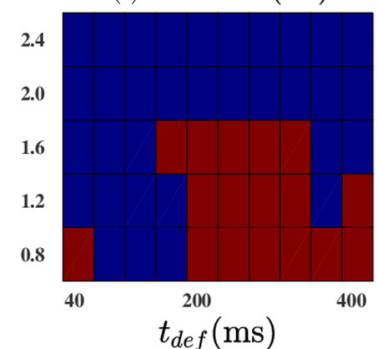

(c) $I_{\text {def }}=15 \mathrm{pA} / \mathrm{pF}$

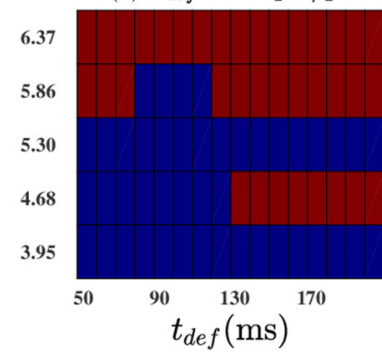

(g) $\sigma=0.68(\mathrm{~cm})$

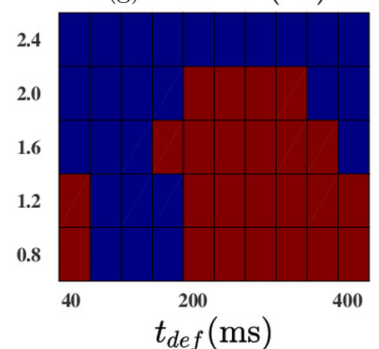

(d) $I_{\text {def }}=20 \mathrm{pA} / \mathrm{pF}$

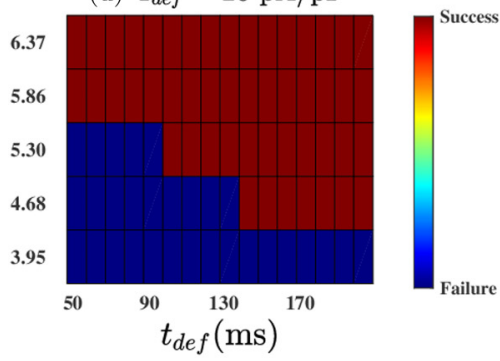

(h) $\sigma=0.73(\mathrm{~cm})$

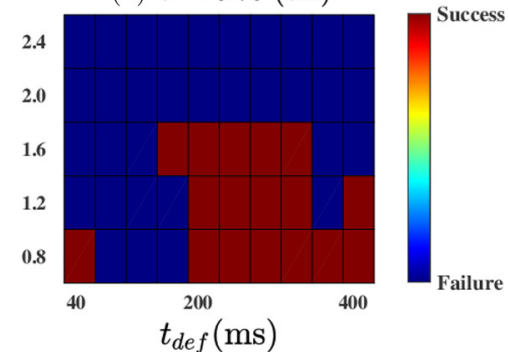

FIG. 9. Pseudocolor plots from (a) to (d) show phase diagrams in the $\left(t_{\text {def }}, \sigma\right)$ plane showing parameter regions in which our Gaussiancontrol scheme succeeds (red) and does not succeed (blue) for different values of the current. Pseudocolor plots from (e) to (h) show another set of phase diagrams from in the $a, t_{\text {def }}$ plane showing parameter regions in which our Gaussian-control scheme succeeds (red) and does not succeed (blue) for different values $\sigma$. 
2D surface of a 3D domain remains a significant challenge [16-18,20].

We provide below stepwise suggestions for the adaptation of our defibrillation scheme to experiments, especially those that study waves of excitation in in vitro cardiac-monolayer experiments:

(i) Data acquisition and heatmap generation:

(a) Spiral waves or broken-spiral waves can be initiated through controlled protocols in cardiac monolayers, e.g., as in Ref. [6].

(b) We use pseudocolor images of $V$ for our $\mathrm{CNN}$, which then generates the heatmap. In experiments, the activationmap image can be used as the input data for our CNN.

(c) Experimental data are noisy. Let us assume that the noisy image is obtained at a time instant $t$. This noisy image can be denoised by using Gaussian or wavelet filters, and then it can be fed into the trained CNN. In turn, this CNN can scan the image, with a square scanning window of side $s$ or with a range of scanning window sizes, and can then output the heatmap. The heatmap is generated at a later instant of time, namely $t+\Delta t$, where $\Delta t$ is the time duration required to generate the heatmap (in our heatmap calculations, $\Delta t \simeq 300 \mathrm{~ms}$ )

(ii) Defibrillation by using the heatmap:

(a) We propose the use of an array of contact electrodes on the layer of cardiac myocytes or tissue (this can be realized as shown in Fig. 1 in Ref. [10] and Fig. 6 in [38]); this can be the experimental analog of the 2D lattice of Gaussians in our defibrillation scheme.

(b) The heatmap can then dictate the strength of the local currents to be applied at these electrodes on the layer of cardiac myocytes.

We hope that our CNN-based detection of spiral waves and our Gaussian-control scheme will be tested in in vitro cardiacmyocyte-monolayer experiments [6-8,15,22,48,49].

Our deep-learning-assisted Gaussian-control method is an important step in the detection and elimination of both broken- and unbroken-spiral waves. Machine-learning techniques have been used, e.g., in Refs. [50-53] for the effective detection of anomalies in electrocardiograms, which can then be eliminated by the controlled delivery of electrical signals via automated defibrillators. No machine-learning method has been employed so far for the detection of spiral waves in, e.g., pseudocolor plots of $V$. Our study uses the complete spatial information in patterns of $V$ to develop an efficient Gaussian-control scheme for the elimination of unbroken- and broken-spiral waves, which are the mathematical analogs of life-threatening VT and VF [16-18].

\section{ACKNOWLEDGMENTS}

We thank DST (IN), CSIR (IN), and UGC (IN) (India) for the support and the Supercomputer Education and Research Centre (SERC, IISc) for computational resources.
[1] R. Mehra, J. Electrocardiol. 40, S118 (2007).

[2] R. Majumder, A. R. Nayak, and R. Pandit, An overview of spiral- and scroll-wave dynamics in mathematical models for cardiac tissue, in Heart Rate and Rhythm: Molecular Basis, Pharmacological Modulation and Clinical Implications, edited by O. N. Tripathi, U. Ravens, and M. C. Sanguinetti (Springer, Berlin, 2011), pp. 269-282.

[3] R. Clayton, O. Bernus, E. Cherry, H. Dierckx, F. H. Fenton, L. Mirabella, A. V. Panfilov, F. B. Sachse, G. Seemann, and H. Zhang, Progr. Biophys. Mol. Biol. 104, 22 (2011).

[4] B. S. Honnekeri, D. Lokhandwala, G. K. Panicker, and Y. Lokhandwala, J. Assoc. Physicians India 62, 36 (2014).

[5] Z.-J. Zheng, J. B. Croft, W. H. Giles, and G. A. Mensah, Circulation 104, 2158 (2001).

[6] J. M. Davidenko, P. F. Kent, D. R. Chialvo, D. C. Michaels, and J. Jalife, Proc. Natl. Acad. Sci. USA 87, 8785 (1990).

[7] T. Ikeda, T. Uchida, D. Hough, J. J. Lee, M. C. Fishbein, W. J. Mandel, P.-S. Chen, and H. S. Karagueuzian, Circulation 94, 1962 (1996).

[8] Z. Y. Lim, B. Maskara, F. Aguel, R. Emokpae, and L. Tung, Circulation 114, 2113 (2006).

[9] I. R. Efimov, V. P. Nikolski, and G. Salama, Circ. Res. 95, 21 (2004).

[10] K. Umapathy, K. Nair, S. Masse, S. Krishnan, J. Rogers, M. P. Nash, and K. Nanthakumar, Circ.: Arrhythmia Electrophysiol. 3, 105 (2010).

[11] J. I. Laughner, F. S. Ng, M. S. Sulkin, R. M. Arthur, and I. R. Efimov, Am. J. Physiol.-Heart Circ. Physiol. 303, H753 (2012).

[12] C. I. Berul, M. J. Aronovitz, P. J. Wang, and M. E. Mendelsohn, Circulation 94, 2641 (1996).
[13] M. Chinushi, D. Kozhevnikov, E. B. Caref, M. Restivo, and N. El-Sherif, J. Cardiovasc. Electrophysiol. 14, 632 (2003).

[14] A. R. Gelzer, M. L. Koller, N. F. Otani, J. J. Fox, M. W. Enyeart, G. J. Hooker, M. L. Riccio, C. R. Bartoli, and R. F. Gilmour, Circulation 118, 1123 (2008).

[15] M. Valderrábano, Y.-H. Kim, M. Yashima, T.-J. Wu, H. S. Karagueuzian, and P.-S. Chen, J. Am. Coll. Cardiol. 36, 2000 (2000).

[16] T. K. Shajahan, S. Sinha, and R. Pandit, Phys. Rev. E 75, 011929 (2007).

[17] T. Shajahan, A. R. Nayak, and R. Pandit, PLoS One 4, e4738 (2009).

[18] A. R. Nayak and R. Pandit, Front. Physiol. 5, 207 (2014).

[19] J. D. Moreno, P.-C. Yang, J. R. Bankston, E. Grandi, D. M. Bers, R. S. Kass, and C. E. Clancy, Circ. Res. 113, e50 (2013).

[20] S. Sinha, A. Pande, and R. Pandit, Phys. Rev. Lett. 86, 3678 (2001).

[21] S. Luther, F. H. Fenton, B. G. Kornreich, A. Squires, P. Bittihn, D. Hornung, M. Zabel, J. Flanders, A. Gladuli, L. Campoy et al., Nature (London) 475, 235 (2011).

[22] B. O. Bingen, M. C. Engels, M. J. Schalij, W. Jangsangthong, Z. Neshati, I. Feola, D. L. Ypey, S. F. Askar, A. V. Panfilov, D. A. Pijnappels et al., Cardiovasc. Res. 104, 194 (2014).

[23] J. Lechleiter, S. Girard, E. Peralta, and D. Clapham, Science 252, 123 (1991).

[24] A. T. Winfree, Science 175, 634 (1972).

[25] M. Falcke, M. Bär, H. Engel, and M. Eiswirth, J. Chem. Phys. 97, 4555 (1992).

[26] R. Imbihl and G. Ertl, Chem. Rev. 95, 697 (1995).

[27] A. Pande and R. Pandit, Phys. Rev. E 61, 6448 (2000). 
[28] F. Marino and G. Giacomelli, Phys. Rev. Lett. 122, 174102 (2019).

[29] J. J. Tyson and J. D. Murray, Development 106, 421 (1989).

[30] J. Rietdorf, F. Siegert, and C. J. Weijer, Dev. Biol. 177, 427 (1996).

[31] D. Barkley, Physica D 49, 61 (1991).

[32] R. R. Aliev and A. V. Panfilov, Chaos Solitons Fractals 7, 293 (1996).

[33] C.-h. Luo and Y. Rudy, Circ. Res. 68, 1501 (1991).

[34] K. H. Ten Tusscher and A. V. Panfilov, Am. J. Physiol.-Heart Circ. Physiol. 291, H1088 (2006).

[35] T. O’Hara, L. Virág, A. Varró, and Y. Rudy, PLoS Comput. Biol. 7, e1002061 (2011).

[36] See Supplemental Material at http://link.aps.org/supplemental/ 10.1103/PhysRevResearch.2.023155 for details such as; (A) the models we use and details about the numerical simulation, (B) parameters varied to generate the datasets, (C) our CNN architecture, and (D) Gaussian-control scheme applied in two other mathematical models for cardiac tissue and an extra figure which explain the reason behind success of our defibrillation scheme.

[37] M. A. Nielsen, Neural Networks and Deep Learning (Determination Press, San Francisco, CA, USA, 2015), Vol. 2018.

[38] W.-J. Rappel and S. M. Narayan, Chaos 23, 023113 (2013).

[39] M.-A. Bray, S.-F. Lin, R. R. Aliev, B. J. Roth, and J. P. Wikswo Jr., J. Cardiovasc. Electrophysiol. 12, 716 (2001).

[40] N. Tomii, M. Yamazaki, T. Arafune, H. Honjo, N. Shibata, and I. Sakuma, IEEE Trans. Biomed. Eng. 63, 1795 (2015).
[41] A. Schlemmer, S. Berg, T. Shajahan, S. Luther, and U. Parlitz, Entropy 17, 950 (2015).

[42] C. M. Ripplinger, Q. Lou, W. Li, J. Hadley, and I. R. Efimov, Heart Rhythm 6, 87 (2009).

[43] S.-J. Woo, J. H. Hong, T. Y. Kim, B. W. Bae, and K. J. Lee, New J. Phys. 10, 015005 (2008).

[44] E. M. Cherry and F. H. Fenton, New J. Phys. 10, 125016 (2008).

[45] G. Huyet, C. Dupont, T. Corriol, and V. Krinsky, Int. J. Bifurcation Chaos 08, 1315 (1998).

[46] P. Bittihn, G. Luther, E. Bodenschatz, V. Krinsky, U. Parlitz, and S. Luther, New J. Phys. 10, 103012 (2008).

[47] D. Hornung, V. Biktashev, N. Otani, T. Shajahan, T. Baig, S. Berg, S. Han, V. Krinsky, and S. Luther, R. Soc. Open Sci. 4, 170024 (2017).

[48] T. Shajahan, S. Berg, S. Luther, V. Krinski, and P. Bittihn, New J. Phys. 18, 043012 (2016).

[49] N. Kudryashova, V. Tsvelaya, K. Agladze, and A. Panfilov, Sci. Rep. 7, 7887 (2017).

[50] C. Figuera, U. Irusta, E. Morgado, E. Aramendi, U. Ayala, L. Wik, J. Kramer-Johansen, T. Eftestøl, and F. Alonso-Atienza, PloS One 11, e0159654 (2016).

[51] L. Maršánová, M. Ronzhina, R. Smíšek, M. Vítek, A. Němcová, L. Smital, and M. Nováková, Sci. Rep. 7, 11239 (2017).

[52] A. Y. Hannun, P. Rajpurkar, M. Haghpanahi, G. H. Tison, C. Bourn, M. P. Turakhia, and A. Y. Ng, Nat. Med. 25, 65 (2019).

[53] S. Singh, S. K. Pandey, U. Pawar, and R. R. Janghel, Proc. Comput. Sci. 132, 1290 (2018). 